I. Boxx, C. Arndt, C.D. Carter, W. Meier

High-speed laser diagnostics for the study of flame dynamics in a lean premixed gas turbine model combustor

Experiments in Fluids, 52:3 pp. 555-567. (2012)

DOI: $10.1007 / \mathrm{s} 00348-010-1022-\mathrm{X}$

Original Publication Available At:

http://link.springer.com/article/10.1007/s00348-010-1022-x

http://dx.doi.org/10.1007/s00348-010-1022-x 


\title{
Highspeed Laser Diagnostics for the Study of Flame Dynamics in a Lean Premixed Gas Turbine Model Combustor
}

\author{
Isaac Boxx ${ }^{1}$, Christoph M. Arndt ${ }^{1}$, Campbell D. Carter ${ }^{2}$, Wolfgang Meier ${ }^{1}$
}

1: Institut für Verbrennungstechnik, Deutsches Zentrum für Luft-und Raumfahrt (DLR), Stuttgart, Germany Isaac.Boxx@dlr.de

2: Air Force Research Laboratory (AFRL) / RZAS. Wright-Patterson AFB, OH. USA

\begin{abstract}
A series of measurements was performed on two technically-premixed, swirl-stabilized methaneair flames (at overall equivalence ratios of $\phi=0.73$ and 0.83 ) in an optically accessible gas turbine model combustor. The primary diagnostics used were combined planar laser-induced fluorescence (PLIF) of the $\mathrm{OH}$ radical and stereoscopic particle image velocimetry (PIV) with simultaneous repetition rates of $10 \mathrm{kHz}$ and a measurement duration of 0.8 seconds. Also measured were acoustic pulsations and $\mathrm{OH}$ chemiluminescence. Analysis revealed strong local periodicity in the thermoacoustically self-excited (or 'noisy') flame $(\phi=0.73)$ in the regions of the flow corresponding to the inner shear layer and the jet-inflow. This periodicity appears to be the result of a helical precessing vortex core (PVC) present in that region of the combustor. The PVC has a precession frequency double (at $570 \mathrm{~Hz}$ ) that of the thermoacoustic pulsation (at $288 \mathrm{~Hz}$ ). A comparison of the various data sets and analysis techniques applied to each flame suggests a strong coupling between the PVC and the thermoacoustic pulsation in the noisy flame. Measurements of the stable ('quiet') flame $(\phi=0.83)$ revealed a global fluctuation in both velocity and heat-release around $364 \mathrm{~Hz}$, but no clear evidence of a PVC.
\end{abstract}




\section{Background}

Gas turbine (GT) relevant flames stabilized by swirl exhibit large effects of flow and flame dynamics like vortex breakdown, precessing helical structures, local flame extinction and/or thermo-acoustic combustion instabilities. Efforts to understand the turbulence-chemistry interactions of combustion in lean premixed and partially-premixed swirl flames in gas turbine combustors has led in recent years to the application of multiple, complementary measuring techniques, in particular 2D laser measuring techniques. The simultaneous application of particle image velocimetry (PIV) and planar laser-induced fluorescence (PLIF) have, for example, contributed to a better understanding of the flow-field/flame interaction in swirl flames [Petersson et al. (2007); Tanahashi et al. (2005); Sadanandan et al. (2008); Stöhr et al. (2009)]. These techniques provide minimally-intrusive measurements in otherwise inaccessible environments such as in high-pressure test rigs and combustion chambers. Research groups have performed laser-based measurements in high-pressure test rigs with optical access in order to investigate GT combustion processes under realistic conditions, i.e. with preheated air, high thermal loads, high turbulence levels and relevant combustor geometries [Lee et al. (2000); Meier et al. (2000); Löfström et al. (2000); Gord et al. (2002); Kojima and Nguyen (2004); Willert et al. (2006); Griebel et al. (2007); Janus et al. (2008); Stopper et al. (2008); Strakey et al. (2008); Ax et al., (2009)]. While these investigations have contributed significantly to a better understanding of certain aspects of GT combustion under pressure, such complex and expensive experiments do not (and, indeed, cannot) result in systematic or comprehensive characterization of swirl flames and are often affected by uncertainties in the boundary conditions, originating, e.g., from limited optical access or a contribution from cooling air to the combustion. High-pressure technical scale (in the MW-range) combustion rigs also degrade the peak performance of most 2D laser measurement techniques through beam-steerage, laser absorption and backscatter, pressure broadening of molecular transitions, fluorescence trapping and decreased $\mathrm{OH}$ concentrations resulting from increased threebody recombination rates. Alternatively, the use of model combustors that can be operated under atmospheric-pressure laboratory conditions has proven to be a good concept to i) gain insight into the interplay between the turbulent swirling flow-field and the flame chemistry and ii) acquire reliable experimental data suitable for model validation.

The relatively low repetition rate (typically $\approx 10 \mathrm{~Hz}$ ) of previously applied planar laser measuring systems such as PLIF of OH radicals and PIV has lead to extensive reliance on series of noncorrelated single-shot images or time-averaged measurements in turbulent flames. In flames with periodic combustion phenomena such as thermoacoustic oscillation, phase-correlated single-shot and averaged measurements have been performed. Extensive databases of high resolution measurements suitable for numerical and code-validation studies have been so acquired. Thorough analysis, in particular using spectral and proper orthogonal decomposition (POD) methods, has reinforced insight gained by these techniques, but, unfortunately, phase-averaged measurements can only isolate mean and statistical cyclic variations of thermo-dynamic and thermo-chemical properties. Information on temporally developing, transient and non-periodic phenomena is lost. Reynolds Averaged Navier-Stokes (RANS) simulations are similarly limited. A fully resolved (temporally and spatially) direct numerical simulation with detailed chemistry and sufficient run duration to be statistically relevant is well beyond the state of the art; furthermore, large eddy simulation (LES) techniques rely on sub-grid scale models that, in many cases, have not been validated under technically relevant operating conditions.

A logical extension of phase-resolved measurement techniques and quasi-steady numerical simulations using techniques such as RANS is the acquisition of long-duration, time-resolved planar measurements of similar flow-field quantities. Although multi-shot planar-LIF imaging with short inter-frame separation has been used extensively by previous researchers [Kychakoff et al. 
(1987); Seitzman et al. (1994); Schefer et al. (1994); Kaminski et al. (1999); Watson et al (2002); Hult et al. (2005); Tanahashi et al. (2005); Tanahashi et al. (2008)], such measurements are of limited use in extracting time-series and frequency characteristics in complex flames. As demonstrated by Upatnieks et al. [2004], Steinberg et al. [2008] and Boxx et al. [2009a, 2009b, 2009c, 2009d], long-duration, highspeed planar measurements allow one to track specific flowfield-flame interactions as they occur, simplifying the interpretation of such data. The acquisition of a statistically significant number of sequential cycles (e.g., several 100) in a single run also renders such interpretations more reliable. Being able to acquire measurements at $\mathrm{kHz}$ rates also results in a short (absolute) acquisition time, a significant advantage in cases where window fouling can be problematic. The present work describes a unique and extremely capable new planar diagnostic system capable of simultaneously acquiring the three-component velocity field and the relative $\mathrm{OH}$ distribution at repetition rates of $10 \mathrm{kHz}$ over measurement durations of $0.8 \mathrm{~s}$. The system was applied to the study of a 25 and a $30 \mathrm{~kW}$, technically premixed, swirl-stabilized methane-air flame in a gas turbine model combustor. One flame burned stably, and the other exhibited strong selfexcited thermoacoustic pulsation.

\section{High Framerate Planar Measurement System}

The $\mathrm{kHz}$ measuring system consists of two independent laser-based measuring systems: a stereoscopic PIV system and a PLIF system configured for $\mathrm{OH}$ detection. Both systems are designed for operation at $10 \mathrm{kHz}$ over 0.8 second durations and have spatial resolution and signal to noise ratios comparable to that of conventional $10 \mathrm{~Hz}$ systems.

\section{$10 \mathrm{kHz}$ PLIF System}

The $10 \mathrm{kHz}$ PLIF system uses a frequency-doubled dye laser (Sirah Credo), pumped with a frequency-doubled, diode-pumped solid state Nd:YAG laser (Edgewave IS-8IIE), which operated at $532 \mathrm{~nm}$ wavelength. At $10 \mathrm{kHz}$ the pump laser delivered $4 \mathrm{~mJ} /$ pulse $(40 \mathrm{~W}$ average output) with $8.5 \mathrm{~ns}$ pulse duration. The active medium of the dye laser was Rhodamine $6 \mathrm{G}$ in Ethanol $(0.09 \mathrm{~g} / \mathrm{L}$ concentration). This dye laser uses a low-threshold resonator cavity optimized for the relatively low pulse energies associated with kHz-rate pump lasers. As a result, this laser is considerably more efficient than the $5 \mathrm{kHz}$ laser used in an earlier configuration of this system (described in Boxx et al., 2009a-d). A $\beta$-barium borate (BBO) crystal, housed in a temperature controlled oven, was used for frequency doubling of the dye laser beam. Isolation of the fundamental and frequency-doubled beams was accomplished with a four-prism beam separator. At $10 \mathrm{kHz}$ pump frequency, the timeaverage output from the dye laser at $283.2 \mathrm{~nm}$ was $\approx 1.4 \mathrm{~W}$ or $0.14 \mathrm{~mJ} / \mathrm{pulse}$. Although higher pulse energies are possible, this comes with an increased risk of damaging either the BBO crystal or the four-prism wavelength separation unit. As such, the laser was operated at $\approx 1.4 \mathrm{~W}$ during the experiments described here.

The $283 \mathrm{~nm}$ beam was formed into a sheet of $\approx 40 \mathrm{~mm}$ (high, in the probe region) using fused silica lenses $\left(f_{\text {plif1 }}=-25 \mathrm{~mm}, f_{\text {plif2 }}=250 \mathrm{~mm}\right)$ in a cylindrical telescope configuration and focused to a waist using a third cylindrical lens $\left(f_{\text {plif3 }}=500 \mathrm{~mm}\right)$. All optics were anti-reflective coated to minimize pulse energy losses before the measurement region. In experiments where PIV was accomplished simultaneously with the OH-PLIF, the $283 \mathrm{~nm}$ laser sheet was overlapped with the PIV laser sheet by transmitting the (532 nm wavelength) PIV sheets through the final 283-nm turning mirror (see Fig. 1a). The PIV and PLIF sheets were overlapped in the near and far fields and also in the probe region. Sheet thicknesses were measured by translating a narrow slit through each beam and using a photodiode to record the spatial distribution; with this approach the $283 \mathrm{~nm}$ laser sheet width was determined to be $\approx 0.4 \mathrm{~mm}$ at the probe volume.

The wavelength tuning of the dye laser to the peak of the isolated $\mathrm{Q}_{1}(7)$ line of the A-X (1-0) transition of $\mathrm{OH}$ was checked daily using a setup consisting of a laminar reference flame, a $10 \mathrm{~cm}$ 
monochromator (Jobin Yvon H10-UV) and a photomultiplier tube for fluorescence detection. The laser line width at $566 \mathrm{~nm}$ was $0.06 \mathrm{~cm}^{-1}$ and thus the value at $283 \mathrm{~nm}$ is expected to be $<0.1 \mathrm{~cm}^{-1}$. The resulting fluorescence emission was acquired with a high frame-rate CMOS camera (LaVision HSS5) and an external, two-stage, lens-coupled intensifier (LaVision HS-IRO). The intensifier uses a $25 \mathrm{~mm}$ diameter S20 photocathode with a P47 phosphor in its first stage and a S20T / P46 for the second (booster) stage. The HSS5 camera has an array size of $1024 \times 1024$ pixels (each 17- $\mu \mathrm{m}$ square) and is digitized to 10-bits. The camera operates in full-frame mode at 3000 frames per second (fps) and possesses $2.6 \mathrm{~GB}$ of on-board memory. This corresponds to approximately $0.8 \mathrm{~s}$ of acquisition time at a $10 \mathrm{kHz}$ frame rate.

Fluorescence from the A-X (1-1) and (0-0) bands was collected at $310 \mathrm{~nm}$ with a fast Cerco $45 \mathrm{~mm}$, $\mathrm{f} / 1.8$ objective lens. Background luminosity was reduced using a short $(200-500 \mathrm{~ns}$, depending on run conditions) intensifier gate. Elastic scattering at $283 \mathrm{~nm}$ was blocked using a high-transmission $(>80 \%$ at $310 \mathrm{~nm})$ bandpass interference filter (Laser-Components $\mathrm{GmbH})$. Flat-field correction for spatial variation of the laser sheet-intensity and other imaging non-uniformities was accomplished using a mean image derived from laser-induced fluorescence of acetone vapor that was doped into the combustion chamber (to be described later). The same intensifier and collection optics were used (separately) with a HSS6 CMOS camera (described below) to also image chemiluminescence emission of the electronically excited $\mathrm{OH}\left(\mathrm{OH}^{*}\right)$. Emission from $\mathrm{OH}^{*}$ is a good qualitative marker of global heat-release [Docquier and Candel (2002), Lee and Santavicca (2003), Hardalupas and Orain (2004)].

\section{$10 \mathrm{kHz}$ PIV System}

The PIV system is based on a dual-cavity, diode-pumped, solid state Nd:YAG laser (Edgewave, IS6IIDE) and a pair of CMOS cameras (LaVision HSS6). The laser produces $2.6 \mathrm{~mJ} /$ pulse at $532 \mathrm{~nm}$ at repetition rates up to $10 \mathrm{kHz}$. Pulse duration is $\approx 14 \mathrm{~ns}$. Pulse timing separation for the PIV system in this study was set to between $\Delta t=5 \& 10 \mu$ s (depending on run conditions), with the $\mathrm{OH}-$ PLIF excitation pulses temporally interlaced between the first and second PIV pulse of the measurement cycle. As with the PLIF system, three cylindrical lenses were used to form the sheet: $f_{\text {piv } 1}=-25 \mathrm{~mm}, f_{\text {piv } 2}=300 \mathrm{~mm}$, and $f_{\text {piv3 }}=1000 \mathrm{~mm}$. To minimize noise arising from inter-frame particle dropout, the beam waist was located somewhat beyond the probe region. The sheet thicknesses were measured in the same manner as for the PLIF beam; at the probe volume, the sheet thicknesses were $\leq 0.7 \mathrm{~mm}$. Mie scattering from titanium dioxide $\left(\mathrm{TiO}_{2}\right)$ particles seeded into the flow was imaged using CMOS cameras mounted on opposite sides of the laser sheet in a forwardscatter imaging configuration. The cameras have a 12-bit, $1024 \times 1024$ pixel imaging arrays $(20 \mu \mathrm{m}$ square pixels) and are capable of imaging at up to $5400 \mathrm{fps}$ in full frame mode. Operated in twoframe PIV mode at $10 \mathrm{kHz}$ (so that the camera frames at $20 \mathrm{kHz}$ ), this array size is reduced to $512 \times$ 512 pixels. The PIV cameras had sufficient on-board memory $(8 \mathrm{~GB})$ to match the acquisition time of the OH-PLIF camera in a given experiment run. Scattered light was collected with $100 \mathrm{~mm}$ macro lenses (Tokina), and image-blur due to off-axis defocusing was corrected using Scheimpflug adaptors between the lenses and cameras. The relatively short integration time $(50 \mu \mathrm{s})$ eliminated the need for interference or coloured-glass filters in front of the lens. Perspective distortion was corrected using a dual-plane, 3D imaging target (LaVision Type 7). The same target was used to map the fields of view of the PIV and PLIF systems to one another. Image mapping, calibration, and particle cross-correlations were completed using a commercial, multi-pass adaptive window offset cross-correlation algorithm (LaVision DaVis 7.2). Final PIV window size and overlap were $16 \times 16$ pixels and $50 \%$, respectively. The field of view for the PIV measurements was $\approx 36 \times$ $36 \mathrm{~mm}$.

\section{Burner and Run Conditions}


The present work focuses on the Turbomeca Gas Turbine Model Combustor (TM-Burner), shown in Fig. 1b. The TM-Burner is a laboratory-scale swirl burner based on an industrial design by Turbomeca S.A. and is operated with a lean $\mathrm{CH}_{4}$-air flame at atmospheric pressure. Dry air at room temperature is fed via a $78 \mathrm{~mm}$ diameter plenum into a central nozzle through 12 radial swirl-vanes. Fuel is injected directly into the swirler through twelve 1-mm diameter holes. The injection of the fuel at high momentum into the air flow at the swirler results in relatively good fuel-air mixing upstream of the optical combustion chamber, and thus this design is referred to as technically premixed. The exit of the burner nozzle has a diameter of $27.85 \mathrm{~mm}$ and has a rounded, conical centerbody. The nozzle is mounted in a combustion chamber with a square, $85 \times 85 \mathrm{~mm}$ cross section. The chamber stands $114 \mathrm{~mm}$ tall and is enclosed by $1.5 \mathrm{~mm}$ thick fused silica window plates, which are supported by posts at each corner. Gas exits the combustion chamber through a thick metal plate with a conical contraction to a $40 \mathrm{~mm}$ diameter exhaust tube. The excellent optical access, compact size and moderate thermal load $(\approx 30 \mathrm{~kW})$ of this burner make both point- and planar-laser measurement techniques feasible (albeit challenging) under laboratory conditions.

Two $\mathrm{CH}_{4}$-air flames were studied in this experiment: a $25 \mathrm{~kW}$ flame with strong self-excited thermo-acoustic pulsations (the 'noisy flame'), and a $30 \mathrm{~kW}$ flame that burns stably and without strong thermo-acoustic pulsation (the 'quiet flame'). These run conditions were chosen to replicate those studied by Meier et al. [2007], designated Flame 1 and Flame 2a, respectively. The Reynolds number of the flame is approximately 35,000, based on cold-flow conditions at the nozzle exit. Meier et al. [2007] estimate the Kolmogorov scale of the flame to be on the order of $0.1 \mathrm{~mm}$, with a corresponding timescale of $35 \mu \mathrm{s}$. This is clearly much smaller than the spatio-temporal resolution of the measuring system, and no attempt is made in this work to analyze the turbulence-chemistry interaction at this scale. The dominant thermoacoustic pulsation, however, (measured at approximately $290 \mathrm{~Hz}$ by Meier et al. [2007]), is well within the temporal resolution of the measurement system.

For each run condition, fuel and air were metered into the burner via electromechanical mass flow controllers. Air was supplied at a rate of $740 \mathrm{~g} / \mathrm{min}$, which was slightly higher than the $734 \mathrm{~g} / \mathrm{min}$ value stated by Meier et al. (2007) but within the accuracy of the flow rate measurement. Approximately $4 \%$ of the air flow was delivered to the burner through a fluidized bed particle seeder. The noisy and quiet flames had equivalence ratios of 0.73 and 0.83 , corresponding to 30 and $36 \mathrm{~g} / \mathrm{min} \mathrm{CH}_{4}$ for the noisy and quiet flame conditions, respectively. Flow-rates of the fuel, seed-air and main-flow air were monitored throughout the experiment via calibration-standard Coriolis mass flowmeters (Siemens Sitrans-FC MassFlo 2100, Model DI-1.5, DI-3 and DI-15, respectively).

Pressure fluctuations in the burner were measured with two microphones (Brüel \& Kjaer, Type 4939): one mounted in a corner post of the combustion chamber and one in the plenum. The microphones were sampled simultaneously with the gate monitor signals of the image intensifiers in order to directly identify the phase of the thermoacoustic cycle for each PLIF and PIV image.

The burner was operated at the run condition for at least twenty minutes prior to each experimental run to allow it to thermally stabilize. During this warm-up period, no seed particles were delivered to the air flow. Shortly before a measurement, the bulk air flow was reduced by approximately $4 \%$ and the same amount was metered into the flow via the particle seeder using a separate electromechanical flow controller. This ensured reliable, reproducible seed density for each run, while minimizing seed-buildup on the combustor windows during the warm-up period.

\section{Results and Discussion}

\section{Mean Flame Characteristics}

Figure 2 shows the mean velocity fields of the noisy and the quiet flames. As expected, the dominant flow structures are a large inner recirculation zone (IRZ), a high velocity in-flow region 
(referred to hereafter as the 'Jet'), separated by the inner shear layer (ISL). Also visible in the mean velocity images is part of the outer recirculation zone (ORZ), which results from the confinement effect of the combustion chamber. Two important features are evident in the mean velocity fields. Consistent with earlier work, the Jet-region of the quiet flame has a significantly wider opening angle than that of the noisy flame. The quiet flame also exhibits a more gradual velocity decay vs. distance and hence a sharper velocity gradient relative to the IRZ. The mean velocity field of the noisy flame shows a more gradual transition between the Jet-region and the IRZ and a more rapid velocity decay along its own length, indicating greater mixing.

Figure $3 \mathrm{a}$ shows the mean $\mathrm{OH}^{*}$ fields of the noisy flame (left side) and the quiet flame (right side). Note the different colourbars for each case. The mean $\mathrm{OH}^{*}$ image for the noisy flame was significantly dimmer (i.e., lower in signal) than that for the quiet flame and extends to peak radial width further downstream of the nozzle. As will be shown later, this is indicative of the strong axial fluctuation in reaction zone location as a result of the thermoacoustic pulsation. Figure $3 \mathrm{~b}$ shows the mean measured OH-PLIF fields for each flame condition. We note that along the centerline at an axial location of approximately $22 \mathrm{~mm}$, a non-uniformity in the sheet-correction image resulted in a non-physical bright spot in the corrected PLIF image. The Jet inflows are identifiable by the large dark regions spreading on either side of the central axis. The darker regions denote a lack of measureable $\mathrm{OH}$ and hence cold $(<1500 \mathrm{~K})$, non-reacted gas. Consistent with the velocity field shown in Fig. 2 and with earlier measurements, it is clear from Fig. $3 b$ that the opening angle of the quiet flame is wider than that for the noisy flame.

Figure 3c shows the mean reaction zone location for each case. In contrast to earlier work (Meier et al. 2007), wherein mean reaction zone location was computed via an Abel deconvolution of the mean line-of-sight integrated $\mathrm{OH}^{*}$ measurements, the mean reaction zone location shown in Fig. $3 \mathrm{c}$ was computed directly from the OH-PLIF measurements. Sadanandan et al. [2008] showed that in a partially premixed swirl flame such as this, the reaction zones may be identified from the regions of highest gradient in OH-PLIF images due to the super-equilibrium $\mathrm{OH}$ concentrations produced there. In this study, the reaction zones were identified as shown in Fig. 4. First, the sheet-corrected OH-PLIF images were binned $(2 \times 2$ pixel $)$ and then smoothed with a $0.6 \mathrm{~mm}$ kernel Gaussian filter. The gradient magnitudes of the resulting images were determined via a Sobel filter. A threshold was then applied to binarize the images and a median filter applied to remove noise artifacts. The direct determination of mean reaction zone location has the advantage of not assuming axi-symmetry as is the case in Abel-deconvolution based method, thus allowing more sophisticated temporal analysis. The results shown in Fig. 3c, however, are consistent with those of Meier et al. [2007]. The reaction zone location of the quiet flame is localized and centered about the ISL region, with little identifiable presence in the IRZ, ORZ or Jet region. The mean flame zone of the noisy flame is much more widely spread, ranging from the region of the Jet, through the ISL and well into the IRZ. A significant amount of wall-interaction is also visible, indicating the reaction zones frequently penetrate past the Jet region. As will be shown later, this is likely as a result of turbulent transport due to large-scale turbulent flow structures.

\section{Time-Dependent Phenomena}

Figure 5 shows the power spectra of axial velocity taken at monitor points within the IRZ, ISL and Jet regions of each flame. The location of each monitor point is shown in the mean velocity images of Fig. 2. The power spectra were computed with 1024-point sample lengths (limited by the memory allocation capability of the analysis software), and the traces represent the average of eight (1024-point) samples collected in a single acquisition run. Also shown in Fig. 5 are power spectra of $\mathrm{OH}^{*}$ emission for each flame, integrated over the combustion chamber. The vertical axes on all spectra are in 'arbitrary units' and thus represent different scales for each trace. Several important characteristics are immediately obvious.

The power spectrum of axial velocity for the quiet flame shows a strong peak at $360-370 \mathrm{~Hz}$ for all 
three monitor points and for the integrated $\mathrm{OH}^{*}$ signal. This indicates that although the flame is quiet, it still has a periodic fluctuation at a characteristic frequency. Given the weakness of the acoustic signal relative to that measured in the noisy flame, this frequency likely represents a damped resonance mode of the combustor for this flame condition. As the spectra uniformly peak at the same frequency at all three spatial locations, the pulsation is not likely indicative of a characteristic fluid-dynamic structure such as a vortex shedding induced by the swirl.

The corresponding power spectra for the noisy flame show starkly different behaviour. All four plotted spectra show strong peaks at approximately $280-290 \mathrm{~Hz}$, which corresponds to the dominant thermo-acoustic oscillation. This peak was also observed in the simultaneously measured acoustic signal. The spectra of integrated $\mathrm{OH}^{*}$ emission and axial velocity in the IRZ show a single peak at this frequency and minor secondary peaks at $560-570 \mathrm{~Hz}$. The spectra for the ISL and Jetregions also show peaks at $560-570 \mathrm{~Hz}$, albeit with the same order of magnitude as those at the thermo-acoustic frequency. These spectra indicate a very strong periodic flow-field phenomenon occurring locally within the ISL and Jet inflow region. The nature of this flow-structure is not immediately clear, given the wide range of fluid-dynamic instabilities known to affect shear-layers in a swirl-stabilized combustor. The lack of a corresponding peak in the spectra of the integrated $\mathrm{OH}^{*}$ signal indicates the phenomenon occurring at $570 \mathrm{~Hz}$ in the ISL and Jet-region does not significantly affect the global heat-release of the flame, which varies at the dominant thermoacoustic pulsation frequency (i.e., 280-290 Hz). The fact that the second peak occurs at twice the thermo-acoustic frequency, however, suggests the periodic flow structure is, in fact, linked somehow to the thermo-acoustic pulsation.

\section{$\underline{P O D \text { Analysis }}$}

In order to identify the fluid-dynamic phenomena responsible for the peaks seen at $570 \mathrm{~Hz}$ in the power spectra of axial velocity, a proper orthogonal decomposition (POD) was performed on the PIV data. POD is a well-established technique in the field of fluid mechanics [Berkooz et al. (1993)]. The result of the POD is a set of orthogonal eigenmodes, representing coherent flow structures ordered by their contribution to the turbulent kinetic energy. For each eigenmode, temporal mode coefficients can be obtained by taking the scalar products of the instantaneous flowfields with the eigenmode. In the present case, the POD was calculated using the method of snapshots [Sirovich (1987)] based on a series of 4096 sequential PIV measurements, acquired at $10 \mathrm{kHz}$.

Plotted in Fig. 6 are the isocontours of vorticity for each of the first three POD eigenmodes for the noisy and the quiet flames, together with the power spectra of the corresponding temporal coefficients. As expected, the dominant energetic modes of each flame are fundamentally different. The eigenmodes of the quiet flame resemble that of a typical symmetric shear layer with standing vortices on either side. In the first mode, the inner vortex structure is centered approximately at $(\mathrm{x}, \mathrm{y})=(12 \mathrm{~mm}, 18 \mathrm{~mm})$. In the second mode, it is centered approximately at $(\mathrm{x}, \mathrm{y})=(10 \mathrm{~mm}$, $10 \mathrm{~mm}$ ). In the third mode, the Jet region is still visible but the side vortices appear to have significantly dissipated. The power spectra of the corresponding temporal modes each show a strong, single peak at $364 \mathrm{~Hz}$. The most reasonable interpretation of the dominant eigenmodes for this case is that they represent a stably-forced shear-layer. The approximate symmetry of vortex structures on either side of the Jet-region indicates that Jet itself is the dominant flow structure in this case and that vortex structures on either side, while periodic in nature, do not significantly affect the flow.

The first two eigenmodes of the noisy flame clearly indicate a vortex-dominated flow-field. The eigenmodes consist of a series of large-scale vortices of opposite rotation aligned along the inner shear-layer. A similar chain of vortices, albeit with smaller diameter and lower vorticity magnitudes, is apparent along the outer shear-layer. In contrast to the quiet flame, there is little sign of the Jet in the first two eigenmodes, suggesting the Jet is strongly affected by the vortices rather 
than vice-versa. The third eigenmode shows little evidence of strong vortical structure, and the corresponding temporal mode shows a dominant peak at approximately $280 \mathrm{~Hz}$. This suggests the third (and thus, less energetic) mode identified in the POD represents the global thermoacoustic pulsation. Figure 7 shows the temporal coefficients for the first three eigenmodes plotted against one another. Whereas modes 1 and 2 show clear similarity in profile, with mode 1 leading mode 2 by approximately one third of a cycle, mode 3 shows little similarity to the other modes. From Fig. 6 we see that the power spectra of the first two temporal modes of the noisy flame are virtually identical, both showing strong fluctuations around 290 and $570 \mathrm{~Hz}$. Considering the similarity in both the frequency content and spatial structure of the first two eigenmodes, it is reasonable to conclude that these two modes represent a single, spatio-temporally transient flow-field structure.

Taken together, the eigenmodes indicate the presence of a helical precessing vortex core (PVC) in the flow-field of the noisy flame. In addition, the eigenmodes indicate that the PVC is the dominant coherent flow structure in this case and that it has a precession frequency of $570 \mathrm{~Hz}$. This finding is reinforced by the numerical simulations of Roux et al. [2005], who predicted the existence of a helical PVC with a precession frequency of $540 \mathrm{~Hz}$ in the same combustor for similar flow-rates, albeit under non-reacting flow conditions. In contradistinction to the simulation of Roux et al. [2005], it does not appear that combustion damps out the PVC in this case. Rather, the PVC remains both present and has a strong influence on both the flame and turbulent flow-field under these conditions. The relatively minor fluctuation observed in the integrated emission from $\mathrm{OH}^{*}$ at $570 \mathrm{~Hz}$, however, indicates that the PVC does not significantly affect the global heat-release of the flame; nonetheless, it may reasonably be expected to have a major influence on local turbulencechemistry interaction and flame dynamics. This possibility is reinforced by Fig. 3c, discussed earlier, wherein the mean reaction zone location is shown to be different for the noisy flame compared to that of the quiet flame.

In order to more closely examine both the nature and the effect of the PVC on local flame dynamics, a series of simultaneous planar velocity and reaction zone measurements are plotted in Fig. 8. Here, the velocity field is reconstructed based on only the first three eigenmodes of the noisy flame. Although using a summation of eigenmodes to represent the flowfield inevitably leads to substantial smoothing of the data, it aids understanding of the effect of the PVC from a phenomenological standpoint. The background contours in this image sequence represent the velocity magnitude. The vectors represent the in-plane components of velocity. Also shown are the contours identified via the OH-PLIF gradient-threshold method described earlier. The image sequence displays every third frame of the $10 \mathrm{kHz}$ measurement sequence, in order to capture approximately one full cycle of the thermoacoustic oscillation (with period of $\approx 3.4 \mathrm{~ms}$ ).

In this image sequence, we see the in-plane component of the PVC structure, together with the coupling mechanism by which it interacts with the thermoacoustic pulsation. In the first frame we see two vortices, the first having positive (out-of-plane) vorticity, centered at $(x, y)=(+15 \mathrm{~mm}$, $+27 \mathrm{~mm}$ ) and the second, having negative (into-plane) vorticity centered at $(-10 \mathrm{~mm}, 13 \mathrm{~mm})$. One also sees an opposed-flow stagnation point at $(5 \mathrm{~mm}, 11 \mathrm{~mm})$, where the inflow with strong positive axial velocity meets negative axial velocity fluid from the IRZ. The reaction zones identified in this measurement are highly fragmented and discontinuous but tend to conform to the passage of these characteristic large-scale flow structures. The reaction zone in frame 1 appears to lie primarily along the periphery of the large-scale vortices identified in the flow, with wrinkles visible near the interaction of the vortices with the stagnation-point flow within the IRZ. At the location of the stagnation point, the reaction zone is somewhat more contiguous than in other regions and tends to align, as one might expect, along the stagnation line between the inflow gases and the IRZ. The conformation of the reaction zone to the passage of large-scale propagating vortices is consistent with similar measurements in a dual-swirl combustor described in Boxx et al. [2009d].

In the measurements following frame 1, we see the two large-scale vortices propagate upward and radially-outward from the centerline. The stagnation point is also seen to move downstream and to 
the right of centerline. In frame 3, we see the appearance of a new vortex to the right of centerline and another stagnation-point flow appear to the left, at $(\mathrm{x}, \mathrm{y})=(-3 \mathrm{~mm}, 7 \mathrm{~mm})$. Both of these then propagate downstream and radially outward from the centerline as before. At the same time, the fragmented and discontinuous reaction zone is seen to move downstream over the first half of the sequence. By frame 6, significant amounts of reaction zone are visible in the IRZ upstream, nearer to the nozzle. Given the sudden (in-plane) appearance and discontinuity of these reaction-zone fragments, it is not immediately clear what physical mechanism is responsible for this upstream appearance of the flame. Given the technically premixed fuel-air inflow, upstream flame propagation and bulk convection induced by the thermo-acoustic pulsation is clearly responsible for some portion of the observed upstream motion of the reaction zones. This motion may appear discontinuous in these planar measurements due to the high through-plane component of velocity. Similar behaviour has been observed in previous studies [Boxx et al. (2009d)]. Given the extensive mixing of post-combustion fluid and technically premixed fuel-air inflow in the IRZ, auto-ignition appears to also be a viable explanation of the observed upstream appearance of reaction zones. Analysis of these two possibilities is ongoing, but a rigorous statistical analysis of each is beyond the scope of this paper.

With the appearance of significant amounts of reaction zone in the upstream region of the IRZ around frame 6, a much longer and more contiguous reaction zone forms in the near nozzle region. This section of reaction zone then begins once more to conform to the passage of large-scale vortices and align with the stagnation point flow in a manner similar to that observed in frame 1 . The velocity field by this point also bears a strong resemblance to that seen in frame 1 , with two diagonally offset, counter-rotating large scale vortices separated by a stagnation-point opposed flow.

Comparing this measurement sequence with the POD results, axial velocity spectra, and mean reaction zone analysis, raises a number of interesting questions regarding the coupling between the PVC with the thermoacoustic pulsation. From the POD analysis, it appears that the dominant flow structures are a helical PVC and a thermoacoustic pulsation. The POD temporal modes and axial velocity spectra indicate this PVC has a characteristic frequency of approximately $570 \mathrm{~Hz}$. The thermoacoustic pulsation is known to be the dominant variable affecting global heat-release and occurs at $\approx 288 \mathrm{~Hz}$, or approximately one half the PVC precession frequency. Inspection of the measurement sequence in Fig. 8 suggests the existence of a periodic axial fluctuation in reaction zone location at approximately the thermoacoustic frequency, evidenced by the similarity in reaction zone structure in the first and last frame of the $0.33 \mathrm{~ms}$ duration sequence. A time-series of $\mathrm{OH}^{*}$ emission integrated over the same region confirm a $288 \mathrm{~Hz}$ fluctuation in heat-release near the nozzle. Over the same period, two large scale vortical structures (those associated with the PVC) propagate through the field of view along the right hand side of the central axis. The first vortex is seen to draw the reaction zone upward and radially outward from the nozzle, allowing the second to enter the combustion chamber absent a contiguous flame-sheet.

Although a rigorous statistical analysis of the $\mathrm{PVC}$, reaction-zone structure, and thermoacoustic oscillation is beyond the scope of the current work, it is clear the three phenomena are closely coupled in the noisy flame. Previous work [Boxx et al. (2009d); Steinberg et al. (2010)] has shown extensive coupling between PVC, reaction zone dynamics and thermoacoustic pulsation in swirlstabilized burners. The fact that the characteristic frequency of the PVC is an integer multiple of the thermoacoustic oscillation suggests a particularly strong coupling in this burner, possibly strengthening the pulsation intensity. What remains to be seen in future analysis, however, is the physical mechanism responsible for this coupling and whether the $570 \mathrm{~Hz}$ frequency of the PVC is a cause or an effect of the $288 \mathrm{~Hz}$ thermoacoustic oscillation. Also yet to be determined is whether the coherent vortex identified in the noisy flame is a single-, or double-helical PVC.

\section{Conclusions}


A series of measurements was performed on two technically premixed, swirl-stabilized $\mathrm{CH}_{4}$-air flames in an optically accessible gas turbine model combustor. The burner (designed by Turbomeca S.A.) was operated with lean mixtures at atmospheric pressure. The two flames described here differed only in fuel flow rate and thus overall equivalence ratio: in one case the equivalence ratio was 0.83 , and the flame was relatively quiet (the 'quiet flame'); in the second case, the equivalence ratio was 0.73 , and the flame exhibited a strong acoustic signature (the 'noisy flame'). The primary diagnostics used were simultaneous planar laser-induced fluorescence (PLIF) of the OH radical and stereoscopic particle image velocimetry (PIV) acquired at repetition rates of $10 \mathrm{kHz}$ over a measurement duration of 0.8 seconds. Also measured were acoustic pulsations and $\mathrm{OH}$ chemiluminescence. Proper orthogonal decomposition (POD) of the PIV time-series measurements was the primary analysis tool and allowed calculation of the dominant energetic modes within each flame.

Analysis revealed a very dynamic and fragmented flame (from the OH PLIF) and strong local periodicity in the thermoacoustically self-excited noisy flame (at about $288 \mathrm{~Hz}$ ) in the regions of the flow corresponding to the inner shear layer and the jet-inflow. This periodicity appears to be the result of a helical precessing vortex core (PVC) present in that region of the combustor. The PVC has a precession frequency double (at about $570 \mathrm{~Hz}$ ) that of the thermoacoustic pulsation. A comparison of the various data sets suggests a strong coupling between the PVC and the thermoacoustic pulsation in the noisy flame. Measurements of the quiet flame revealed a global fluctuation in both velocity and heat-release around $364 \mathrm{~Hz}$ but no clear evidence of a PVC.

\section{Acknowledgments}

C. Carter acknowledges support of the Air Force Office of Scientific Research (AFOSR) Windows on Europe Program.

Figure 1a is reproduced with permission from Springer-Verlag - from Sustained multi-kHz flamefront and 3-component velocity-field measurements, by I.Boxx, M. Stöhr, C. Carter and W. Meier. Appl Phys B (2009) 95: 23-29. and from Elsevier Science - from Temporally resolved planar measurements of transient phenomena in a partially pre-mixed swirl flame in a gas turbine model combustor, by I.Boxx, M. Stöhr, C. Carter and W. Meier. Combustion and Flame, 157 (2009), 1510-1525

Figure $1 \mathrm{~b}$ is reproduced with permission from Elsevier Science - from Detailed characterization of the dynamics of thermoacoustic pulsations in a lean premixed swirl flame, by W. Meier, P. Weigand, X.R. Duan, R. Giezendanner-Thoben. Combustion and Flame 150 (2007) 2-26.

\section{References}

Ax H, Stopper U, Meier W, Aigner M, Güthe F (2009) Experimental analysis of the combustion behaviour of a gas turbine burner by laser measurement techniques. Proc. ASME Turbo Expo, GT2009-59171.

Berkooz G, Holmes P, Lumley JL (1993) The Proper Orthogonal Decomposition in the Analysis of Turbulent Flows. Ann. Rev. Fluid Mech. 539-575.

Boxx I, Stöhr M, Blumenthal R, Carter C, Meier W (2009a) Investigation of a gas turbine model combustor by means of high-speed laser imaging. 47th AIAA Aerospace Sciences Meeting, AIAA 2009-644.

Boxx I, Heeger C, Gordon R, Böhm B, Dreizler A, Meier W (2009b) On the importance of temporal context in interpretation of flame discontinuities. Combust. Flame, 156:269-271.

Boxx I, Stöhr M, Carter C, Meier W (2009c) Sustained multi-kHz flamefront and 3-component velocity-field measurements for the study of turbulent flames. Appl. Phys B, 95(1) 23-29. 
Boxx I, Stöhr M, Carter C, Meier W (2009d) Temporally resolved planar measurements of transient phenomena in a partially pre-mixed swirl flame in a gas turbine model combustor. Comb. Flame, Volume: 157:1510-1525.

Docquier N, Candel S (2002), Combustion control and sensors: a review. Prog. Energy Combust. Sci. 28:107-150.

Gord JR, Brown MS, Meyer TR (2002) Optical diagnostics for characterizing advanced combustors and pulsed-detonation engines. 22nd AIAA Aerodynamic Measurement Technology and Ground Testing Conference, AIAA 2002-3039.

Griebel P, Siewert P, Jansohn P (2007) Flame characteristics of turbulent lean premixed methane/air flames at high pressure: Turbulent flame speed and flame brush thickness. Proc. Combust. Inst. 31:3083-3090.

Hardalupas, Y., Orain, M. (2004), "Local Measurement of the Time-Dependent Heat Release Rate and Equivalence Ratio Using Chemiluminescence Emissions from a Flame”, Combust. Flame 139: 188-207.

Hult J, Meier U, Meier W, Harvey A, Kaminski C.F., Proc. Combust. Inst. 30 (2005) 701-709.

Kaminski CF, Hult J, Alden M (1999) Appl Phys B 68:757-760

Kojima J, Nguyen QV (2004) Measurement and simulation of spontaneous Raman scattering in high-pressure fuel-rich H2-air flames. Meas. Sci. Technol. 15:565-580.

Kychakoff G, Paul P.H., Cruyningen I, and Hanson R.K. (1987) Movies and 3-D images of flowfields using planar laser-induced fluorescence. App. Opt. 26:2498-2500

Janus B, Dreizler A, Janicka J (2007) Experiments on swirl stabilized non-premixed natural gas flames in a model gas turbine combustor. Proc. Comb. Inst. 31:3091-3098.

Lee SY, Seo S, Broda JC, Pal S, Santoro RJ (2000) An experimental estimation of mean reaction rate and flame structure during combustion instability in a lean premixed gas turbine combustor. Proc. Combust. Inst. 28:775-782.

Lee, J.G., and Santavicca, D.A. (2003), "Experimental Diagnostics for the Study of Combustion Instabilities in Lean Premixed Combustors", J. Propulsion and Power 19:735-750.

C. Löfström, et al. (2000) Feasibility studies and application of laser/optical diagnostics for characterisation of a practical low-emission gas turbine combustor. Proc. ASME Turbo Expo, 2000GT-0124

Meier UE, Wolff-Gaßmann D, Stricker W (2000) LIF imaging and 2D temperature mapping in a model combustor at elevated pressure. Aerosp. Sci. Technol. 4:403-414.

Meier W, Weigand P, Duan XR, Giezendanner-Thoben R (2007) Detailed characterization of the dynamics of thermoacoustic pulsations in a lean premixed swirl flame. Combust. Flame 150:2-26.

Petersson P, et al. (2007) Simultaneous PIV_OH-PLIF, Rayleigh thermometry_OH-PLIF and stereo PIV measurements in a low-swirl flame. Appl. Opt. 46:3928-3936.

Roux S, Lartigue G, Poinsot T, Meier U, Berat C (2005) Studies of mean and unsteady flow in a swirled combustor using experiments, acoustic analysis, and large eddy simulations. Combust. Flame 141:40-54.

Sadanandan R, Stöhr M, Meier W (2008) Simultaneous OH-PLIF and PIV measurements in a gas turbine model combustor. Appl. Phys. B 90:609-618.

Schefer RW, Namazian M, Filtopoulos EEJ, Kelly J (1994) Proc Combust Inst. 25:1223-1231

Seitzman JM, Miller MF, Island TC, Hanson RK (1994) Proc Combust Inst 25:1743-1750

Sirovich L (1987) Turbulence and the dynamics of coherent structures. Q. Applied Mathematics. XLV 3:561-571.

Steinberg AM, Driscoll JF, Ceccio SL (2008) Measurements of turbulent premixed flame dynamics 
using cinema stereoscopic PIV. Exp Fluids 44:985-999.

Steinberg AM, Boxx I, Stöhr M, Carter C, Meier W. (2010) "Flow-flame interactions causing acoustically coupled heat release fluctuations in a thermo-acoustically unstable gas turbine model combustor", Combustion and Flame. In Press, doi:10.1016/j.combustflame.2010.07.011

Stöhr M, Sadanandan R, Meier W (2009) Experimental study of unsteady flame structures of an oscillating swirl flame in a gas turbine model combustor. Proc. Comb. Inst. 32:2925-2932.

U. Stopper, M. Aigner, W. Meier, R. Sadanandan, M. Stöhr, I.S. Kim (2008) Flow field and combustion characterization of premixed gas turbine flames by planar laser techniques. Proc. ASME Turbo Expo, GT2008-50520.

Strakey PA, Woodruff SD, Williams TC, Schefer RW (2008) OH-Planar Fluorescence Measurements of Pressurized, Hydrogen Premixed Flames in the SimVal Combustor. AIAA J. 46:1604-1613.

Tanahashi M, Murakami S, Choi GM, Fukuchi Y, Miyauchi T (2005) Simultaneous CH-OH PLIF and stereoscopic PIV measurements of turbulent premixed flames. Proc. Combust. Inst. 30:16651672.

Tanahashi M., Taka S., Shimura M., Miyauchi T., (2008) CH double-pulsed PLIF measurement in turbulent premixed flame. Exp Fluids 45:323-332

Upatnieks A, Driscoll JF, Rasmussen CC, Ceccio SL (2004) Liftoff of turbulent jet flames Assessment of edge flame and other concepts using cinema-PIV. Comb. Flame 138:259-272.

Watson K.A., Lyons K.M., Carter C.D. Donbar J.M. (2002) Simultaneous two-shot CH planar laser-induced fluorescence and particle image velocimetry measurements in lifted CH4/air diffusion flames. Proc. Combust. Inst., 29:1905-1912.

C. Willert, C. Hassa, G. Stockhausen, M. Jarius, M. Voges, J. Klinner (2006) Combines PIV and DGV applied to a pressurized gas turbine combustor facility. Meas. Sci. Technol. 17:1670-1679. 


\section{Figure Captions}

Figure 1. Experimental Setup

Fig. 2. Mean Velocity Field. Above - Noisy Flame. Below Quiet Flame

Fig. 3. Mean fields of a) $\mathrm{OH}^{*}$ emission b) $\mathrm{OH}$ PLIF c) Reaction Zone. Left side- Noisy flame, Right Side-Quiet flame. The colourbar for a.) refers directly to mean measured signal counts. Those for b.) and c.) denote arbitrary units on a common scale for each.

Fig. 4. Image processing steps used to determine reaction zone location

Fig. 5. Power spectra of axial velocity and integrated $\mathrm{OH}^{*}$ emission.

Fig. 6. POD Eigenmodes of the noisy and quiet flame velocity field measurements

Fig. 7. Temporal mode coefficients for the first three POD eigenmodes of the noisy flame velocity field.

Fig. 8. Measurement sequence showing interaction of the reaction zone and PVC flow-structure in the noisy flame. The sequence represents approximately one full cycle of the thermoacoustic oscillation. Every third measured frame is plotted here. 


\section{Figures}

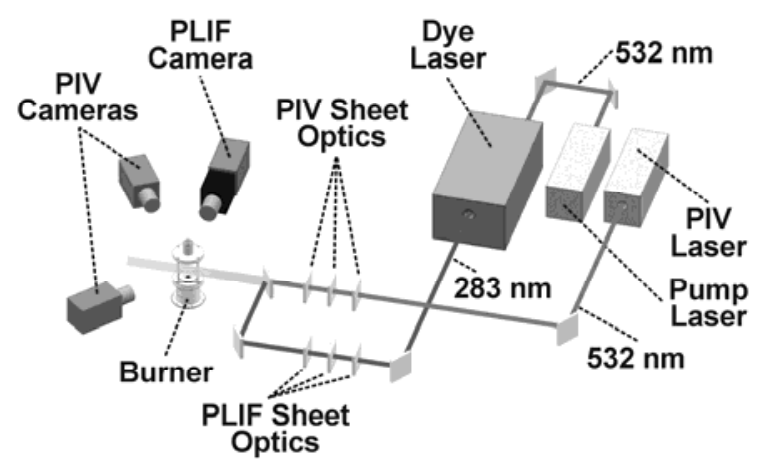

a)

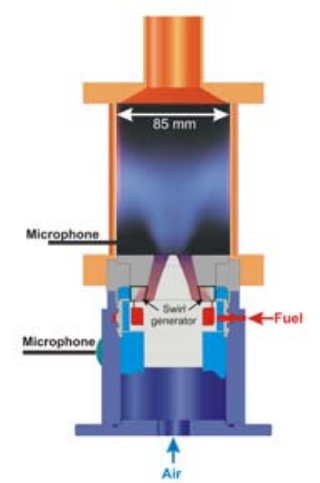

b)

Figure 1. Experimental Setup 


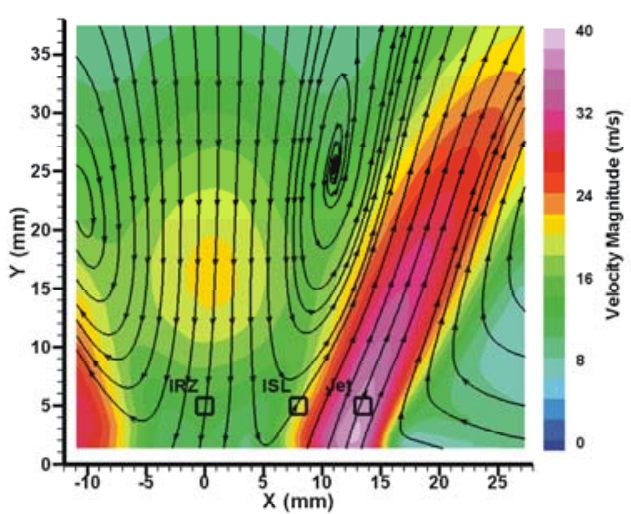

Noisy Flame

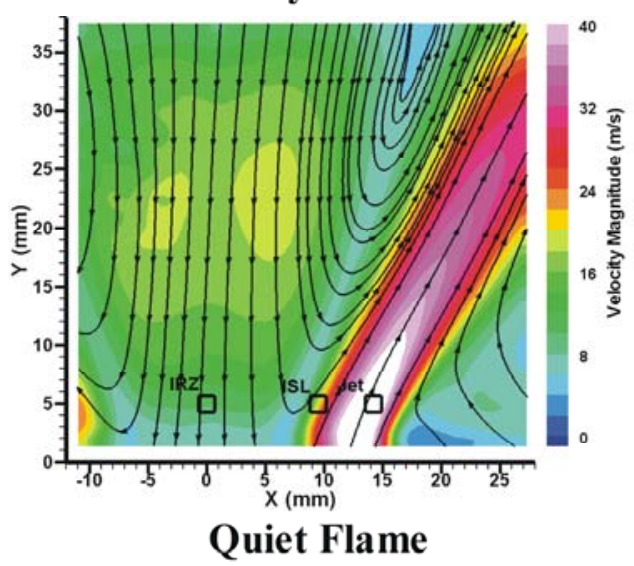

Figure 2. Mean Velocity Field. Above - Noisy Flame. Below Quiet Flame 


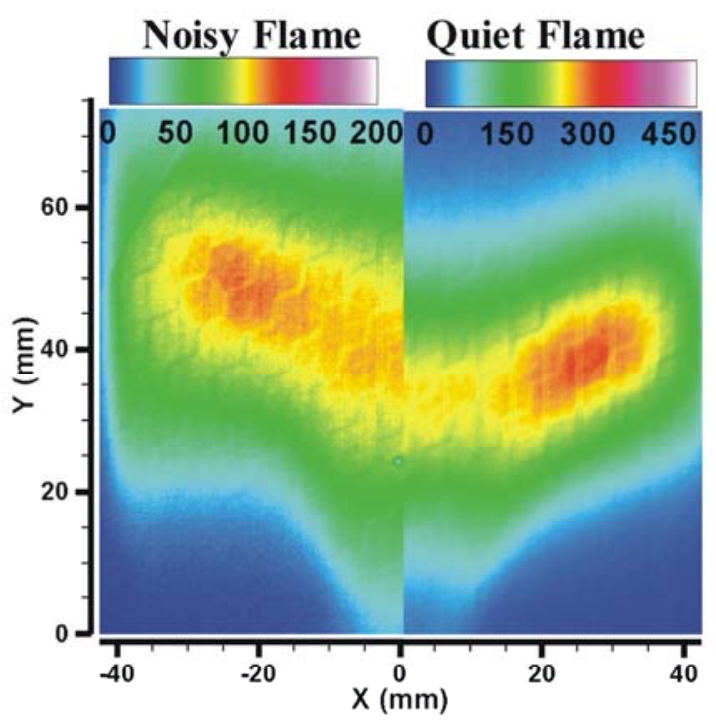

$\mathrm{OH}^{*}$ Chemiluminescence

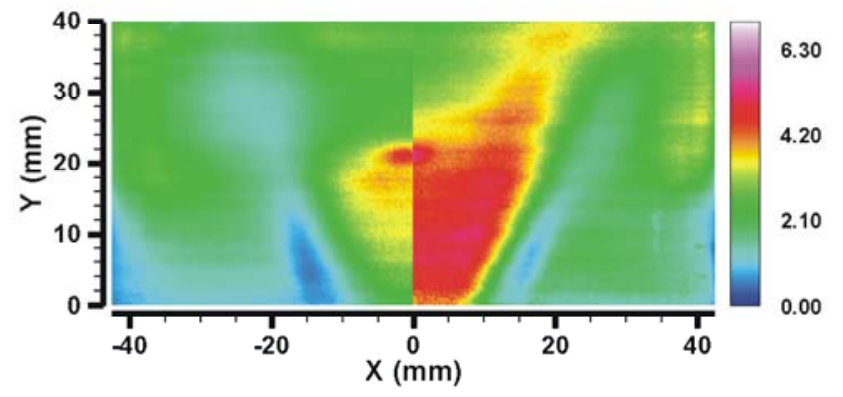

OH-PLIF

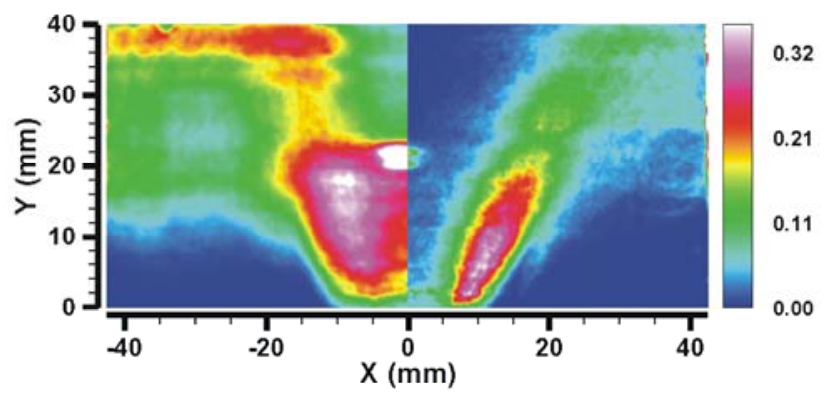

Reaction Zone

Fig. 3. Mean fields of a) $\mathrm{OH}^{*}$ emission b) $\mathrm{OH}$ PLIF c) Reaction Zone. Left side- Noisy flame, Right Side-Quiet flame. The colourbar for a.) refers directly to mean measured signal counts. Those for b.) and c.) denote arbitrary units on a common scale for each. 

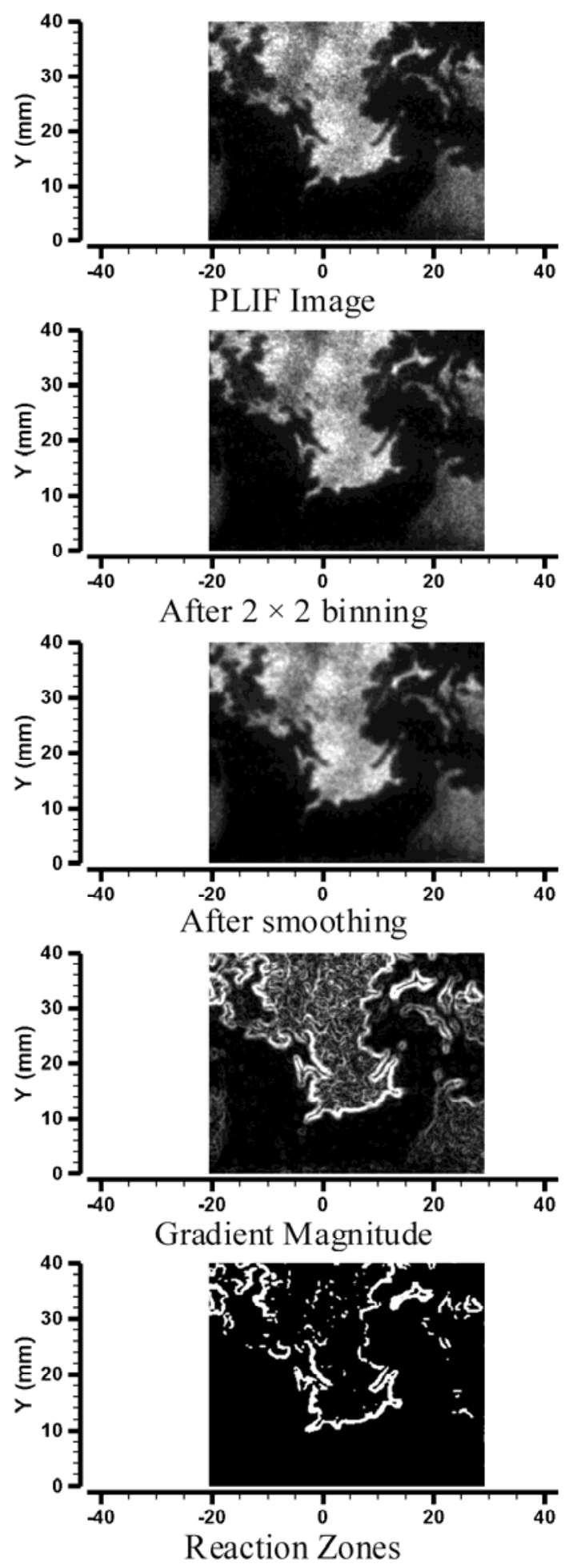

Fig. 4. Image processing steps used to determine reaction zone location 


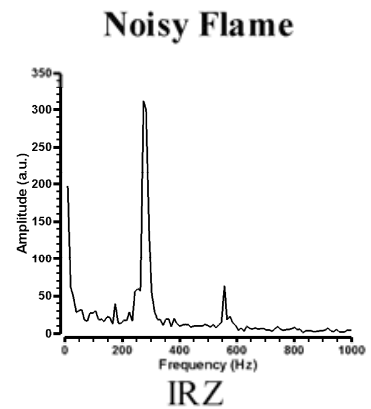

Quiet Flame

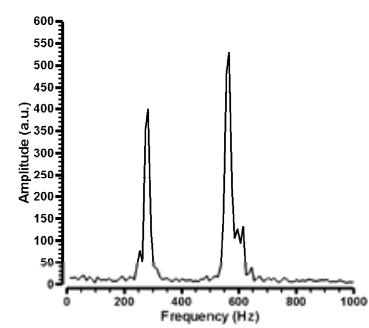

ISL

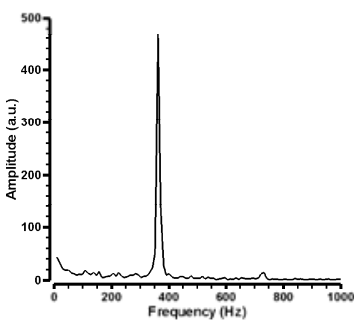

IRZ

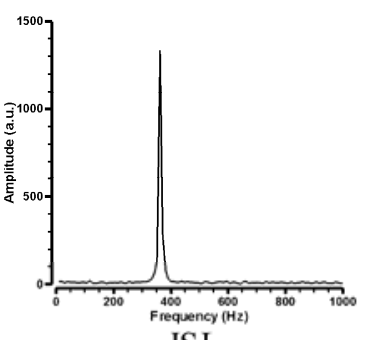

ISL
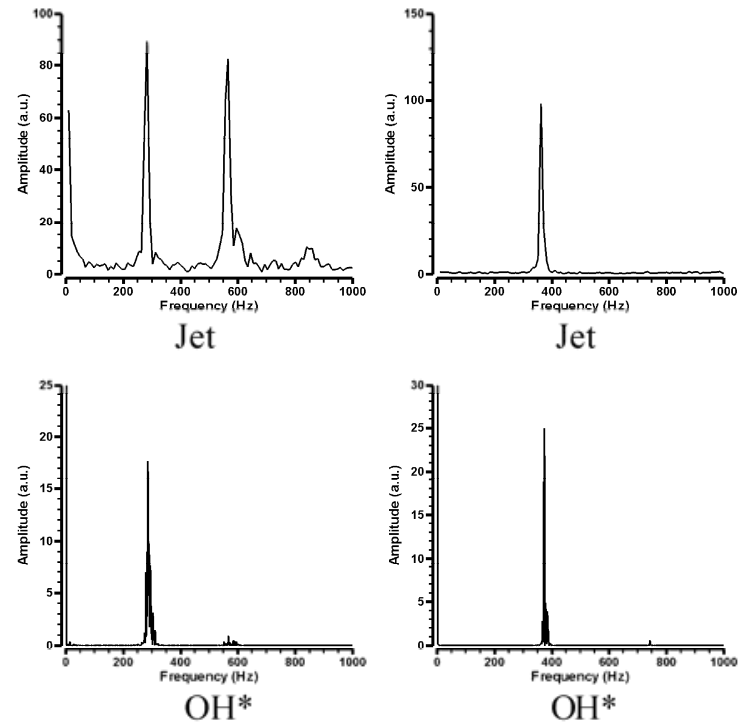

Fig. 5. Power spectra of axial velocity and integrated $\mathrm{OH}^{*}$ emission. 

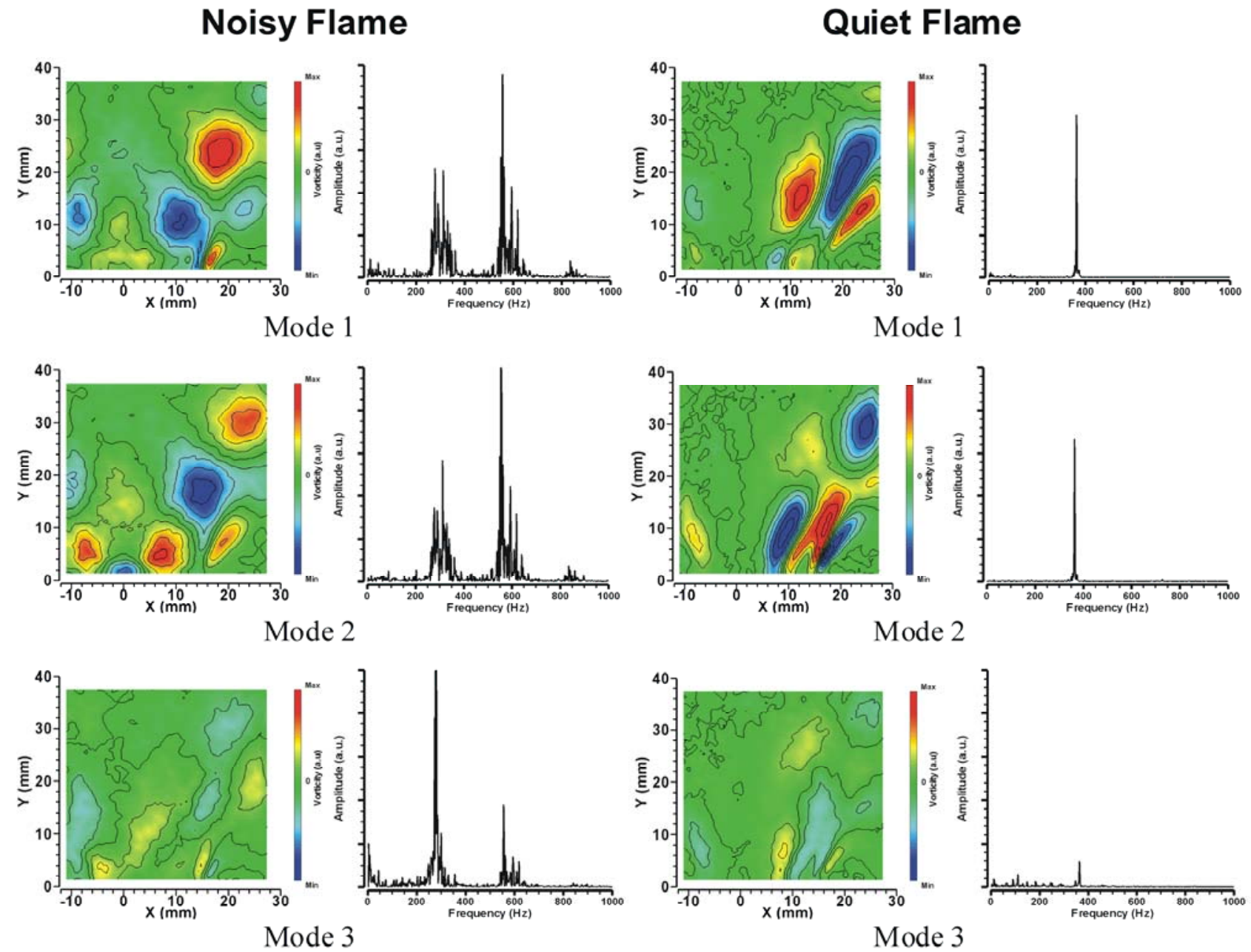

Fig. 6. POD Eigenmodes of the noisy and quiet flame velocity field measurements 


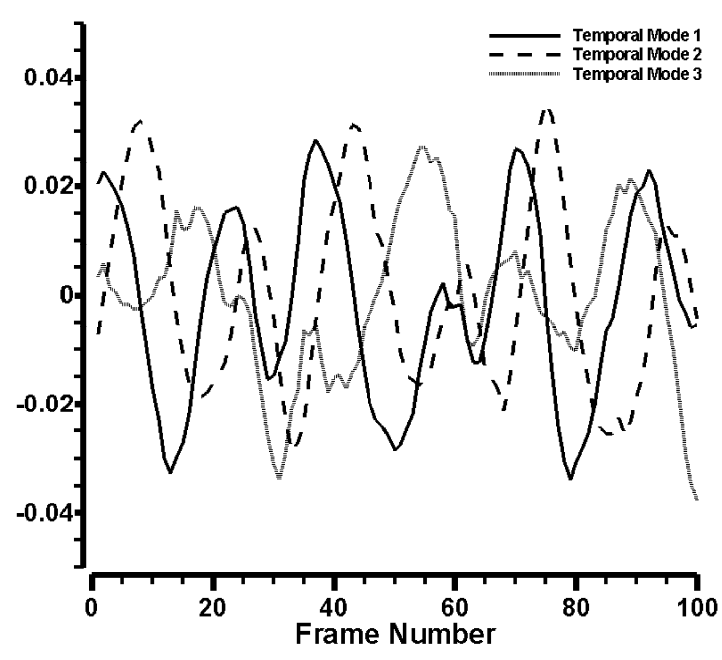

Fig. 7. Temporal mode coefficients for the first three POD eigenmodes of the noisy flame velocity field. 

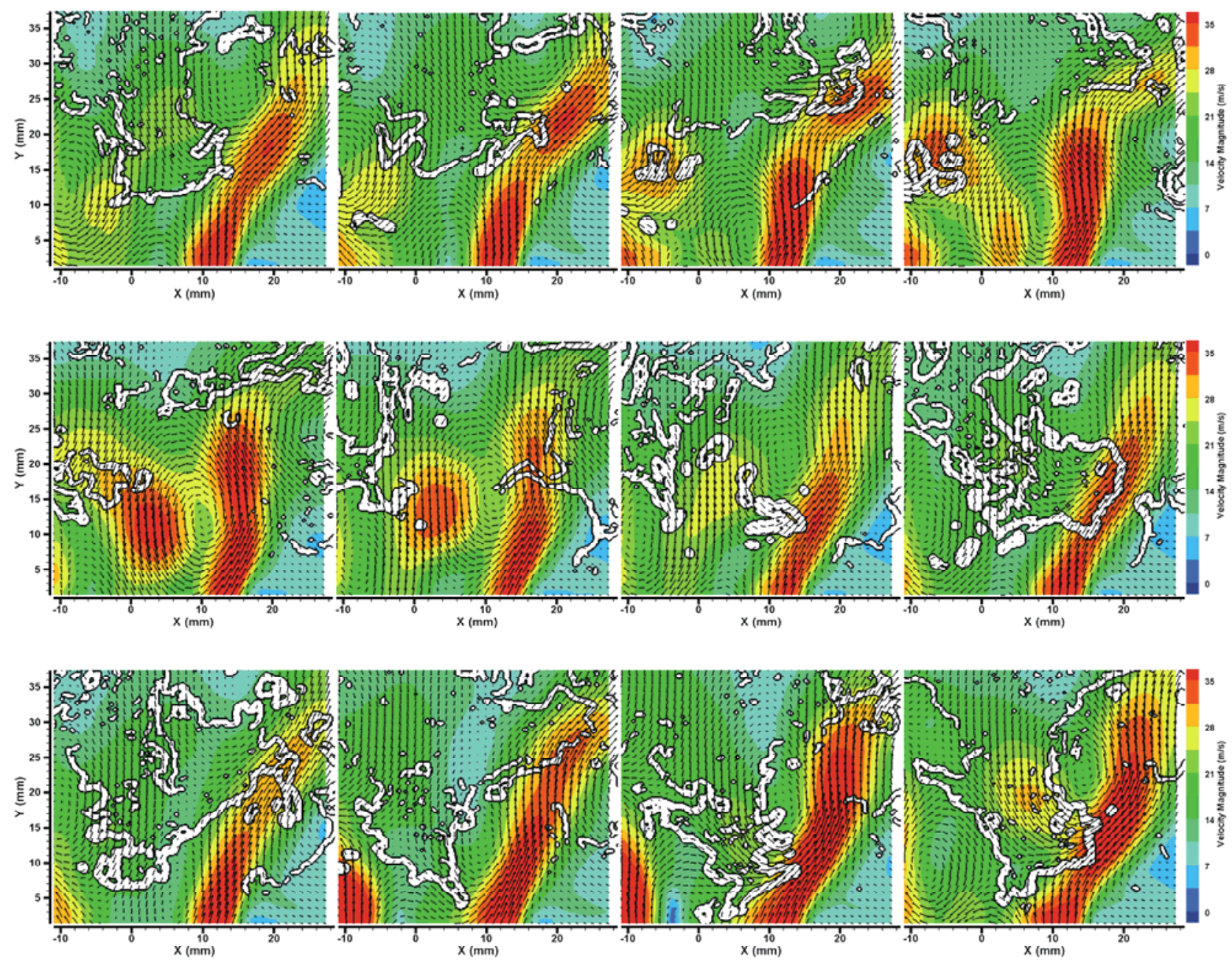

Fig. 8. Measurement sequence showing interaction of the reaction zone and PVC flow-structure in the noisy flame. The sequence represents approximately one full cycle of the thermoacoustic oscillation. Every third measured frame is plotted here. 